\title{
Salpingo-oophorectomy specimens for endometrial cancer staging: a comparative analysis of representative sampling versus whole tissue processing
}

\author{
Oluwole Fadare, $\mathbf{M D}^{1,2}$ and Dineo Khabele, $\mathbf{M D}^{2,3}$ \\ ${ }^{1}$ Department of Pathology, Microbiology and Immunology, Vanderbilt University School of \\ Medicine, Nashville, TN \\ ${ }^{2}$ Department of Obstetrics and Gynecology, Vanderbilt University School of Medicine, Nashville, \\ TN \\ ${ }^{3}$ Department of Cancer Biology, Vanderbilt University School of Medicine, Nashville, TN
}

\begin{abstract}
Involvement of the ovary and/or fallopian tube by an endometrial cancer is uncommon but clinically significant, since this is one of the indications for adjuvant chemotherapy. The authors evaluated whether the routine microscopic evaluation of the adnexal organs in this setting should be of the entire specimen, or of representative sections. Slides and reports were reviewed for 105 consecutive patients that underwent a staging salpingo-oophorectomy (205 ovaries, 210 tubes) for endometrial carcinoma/carcinosarcoma. The study period encompassed the periods before and after an institutional policy change from discretionary (predominantly representative) adnexal sampling to obligatory total processing. 94 and 111 ovaries (and 92 and 118 tubes) were entirely and representatively processed respectively. Even when using the most expansive definition of ovarian gross abnormality (definition with the highest sensitivity and lowest specificity for microscopically-confirmed cancer), we still identified 4 (of 148: $2.7 \%$ ) grossly normal ovaries and 3 (of $187: 1.6 \%$ ) grossly normal tubes that were found to harbor microscopic cancers. There was no significant increase in the number of grossly occult cancers detected after the policy change, and $5(71 \%)$ of the 7 grossly occult cancers were in the representatively-sampled group. Approximately 3.76 more blocks per patient were required for total over representative processing, and the total cost of these additional sections was estimated to be $\$ 25.57$ per patient. In conclusion, the 1.6-2.7\% of grossly normal adnexa that proved to be cancerous represents, at least theoretically, the risk for misdiagnosis and understaging that is associated with representative sampling, at relatively modest savings. However, the findings in this study do not provide direct evidentiary support for routine complete processing.
\end{abstract}

\section{INTRODUCTION}

One of the contributions that surgical pathologists make to the care of cancer patients is the accurate assessment and documentation of the extent of cancer spread in their resected

Correspondence Oluwole Fadare, MD Department of Pathology, Microbiology and Immunology Vanderbilt University Medical Center $116121^{\text {st }}$ Avenue S, Nashville, TN 37232 oluwolefadare@yahoo.com. 
organs. At present, the gold standard for making this assessment is microscopic examination of selected tissue sections as directed and informed by a trained prosector's assessment of macroscopic aberrations. This time honored practice has proven to be an effective way of striking an appropriate balance between the need to accurately document the stage of disease and the need to keep pathology practice cost-efficient by avoiding microscopic examination of all submitted tissues. However, in resections wherein additional organs are routinely submitted with the primary, cancer-bearing organ, sampling issues of the former arise. For multiple organ resections where the second organ was removed for anatomic continuity and technical factors (such as the splenectomies for distal pancreatectomies, or appendectomies for cecal cancers), the traditional approach to sampling these additional organs has proven to be effective. In composite resections wherein the additional organs are removed for anatomic continuity as well as being a recognized destination for early metastatic disease, the degree of sampling for these organs may be controversial. In specimens from radical prostatectomies for prostate cancer, for example, consensus has recently emerged that the routine blocking of the seminal vesicles is unnecessary (1). No such consensus exists with many other comparable organs.

With 47,130 new cases diagnosed every year in the United States, endometrial carcinoma is most common gynecologic tract malignancy, and the $4^{\text {th }}$ most commonly diagnosed noncutaneous malignancy in women (2). The vast majority of endometrial cancer patients have disease that is confined to the uterus (International Federation of Gynecologists and Obstetricians [FIGO] stage I or II) at diagnosis (3). This can, however, only be definitively ascertained after surgical staging (which entails, at minimum, a total hysterectomy, salpingo-oophorectomy and regional lymphadenectomy) and the pathologic assessment of the resultant organs (4). Involvement of the ovaries and/or fallopian tubes (adnexa) by endometrial cancer is indicative of stage IIIA disease under the FIGO staging system (3), and adnexal involvement is one of the indications for adjuvant chemotherapy in patients with endometrial cancer (5). This confluence of factors, i.e. a relatively common cancer, an uncommon event (adnexal involvement by endometrial cancer) and the immensity of its clinical significance when present, underscore the importance of the nature of pathologic review to ascertain the event, and the cost-effectiveness of that review. The purpose of this study is to assess whether the routine microscopic evaluation of the adnexa, especially when they are grossly normal, should always be of the entire specimen, or of representative sections.

\section{MATERIALS AND METHODS}

\section{Case Selection}

After approval from our institutional review board, the pathology database at Vanderbilt University Medical Center was queried for all endometrial carcinomas and carcinosarcomas that were surgically resected between September 2009 and August 2011. The mid point of this 2-year period coincided with an institutional policy change in how the ovaries and fallopian tubes are processed in endometrial cancer-related resections. Prior to this change, whether the adnexal organs were submitted completely or representatively was entirely at the discretion of the individual prosector. The policy change mandated the submission of the 
entire adnexal organs in all endometrial cancer-related resections. The final dataset only included cases that were resected with a total hysterectomy and at minimum, a unilateral salpingo-oophorectomy. Cases resected with simple hysterectomies only were excluded. After review of pathologic reports and slides, the following items of clinicopathologic information were documented for each case: 1) patient age and disease stage per FIGO criteria, 2) Dimensions for each ovary and fallopian tube, 3) Number of tissue blocks processed for each ovary and fallopian tube, 4) Any gross abnormalities of the adnexal organs as indicated in their gross descriptions (see below), 5) Adnexal sampling methodology (representative sampling versus complete tissue processing), 6) Size of metastatic lesions in the adnexa (from the gross descriptions or the slides in cases where the metastatic lesion was microscopic), 7) Tumor-related information, including histotype.

\section{Assessment of Gross Abnormalities of the Ovary and Fallopian Tube}

For each ovary and fallopian tube, we documented the presence or absence of specific gross abnormalities, as discerned from their gross descriptions, and as supplemented with specimen photographs when available. Gross abnormalities for the ovary included: 1) enlargement; 2) any cystic change; 3) mass lesion/nodules; and 4) hemorrhage or nonspecific softening. Cystic change was further classified into "simple" cystic change (devoid of internal [intracystic] papillations or nodules) and "complex" cystic change (with internal papillations and/or nodules). An ovary was considered to be enlarged if its maximal linear dimension was greater than $4 \mathrm{~cm}$. The latter figure was estimated as follows: The maximal dimension of the post-menarchal ovary is typically in the $2.5 \mathrm{~cm}$ to $5 \mathrm{~cm}$ range (mean volume $8 \mathrm{~cm}^{3}$, range $2.5-20 \mathrm{~cm}^{3}$ ). (6-8). In postmenopausal, healthy females, the mean normal ovarian size, as assessed by ultrasound, is $2.2 \mathrm{~cm}$ (9). In one assessment of 183 normal postmenopausal ovaries, the maximal length was $3 \mathrm{~cm}$ (10). Given the objectives of the current study, we selected a size threshold of $4 \mathrm{~cm}$ that was most likely to capture abnormalities in both postmenopausal and premenopausal populations. For the fallopian tube, only 3 parameters were recorded: 1) any cystic change,; 2)hemorrhage/non-specific softening; and 3) mass lesion/nodules.

\section{Accuracy and Related Parameters}

For each ovary and fallopian tube, involvement by cancer, as determined microscopically "confirmed cancer" -, was assigned as the gold standard against which the performance of individual gross abnormalities were measured. Using that standard, the sensitivity, specificity, accuracy, positive predictive value and negative predictive value for each gross abnormality (and combination of abnormalities) in predicting confirmed cancer was determined. In these analyses, a case was considered a "true positive" for a given gross abnormality if that abnormality was present as along with confirmed cancer. A true negative lacked both the gross abnormality and confirmed cancer. A false positive had the gross abnormality but no confirmed cancer, and a false negative was devoid of the gross abnormality, but had confirmed cancer.

\section{Cost Analysis}

The cost of every additional section of tissue was determined by combining the cost of processing the block and the cost of a physician evaluating the resultant slides. We used 
productivity standards for histology laboratories as had previously been reported by Buesa (11). The author estimated the total time value per unit for grossing (4.3 minutes), cassetting (1.1 minutes), embedding (1.2 minutes), slide labeling (20 seconds), preparing blocks to cut (23 seconds), block trimming ( 25 seconds), microtome cutting ( 2.5 minutes), hematoxyin/ eosin staining (1.2 minutes), and slide coverslipping (45 seconds), to be approximately 12.18 minutes (11). Given the automated nature of many of the functions in our histopathology laboratory, and the multitasking that is intrinsic to our work systems, that figure was reduced to 10 minutes. The average annual salary for a staff-level histotechnologist (United States, 2010) is approximately \$54,080/year (\$26 per hour) (12). This translates, according to the relevant system from the American Medical Association's Specialty Society Relative Value Scale Update Committee (13), to approximately $\$ 0.43$ / minute (14). Since grossing may be performed by pathology assistants (whose salaries typically exceed those of a histotechnologist), a resident (whose salaries are generally comparable to those of a histotechnologist) or a pathology technician (whose salaries are typically less than those of a histotechnologist), we elected to use the salary figures for a histotechnologist in our calculations for the prosector. Therefore, the labor-related cost of each block prior to review by a physician was conservatively estimated to be $\$ 4.30 /$ block. The total materials related cost to process one block was estimated to be $\$ 0.3614$. This figure was generated by analyzing the total cost of reagents that were used in our processor in a random month, and taking into account the number of blocks generated and the cost of waste removal. The total materials related cost to generate one H\&E stained slide was estimated to be $\$ 0.5322$. This figure was generated from an analysis of the yearly reagents cost for the H\&E stainer, the total number of $H \& E$ slides that are generated, slide and labeling costs, and costs associated with waste removal. The total materials $(\$ 0.36+\$ 0.53)$ and labor (\$4.30) related costs to generate one H\&E slide before review by a physician was thus estimated to be $\$ 5.1936$

The average time it would take for a pathologist to evaluate one additional slide from the ovary or fallopian tube was estimated to be 1 minute. This figure is somewhat arbitrary, and was based on the experiences of one of the authors. For pathologist compensation estimates, we used figures from Nash et al (15). In that study, the average total annual compensation for pathologists was $\$ 231,250$, with a median work week of 49 hours, yielding an estimated compensation of $\$ 1.51$ per minute (15). The aforementioned annual salary is at the conservative end of another survey, which found that the annual salary for pathologists typically ranged from $\$ 239,000$ to $\$ 331,842$ (16).

\section{RESULTS}

Specimens from a total of 105 consecutive patients were studied. Clinicopathologic information for these patients is outlined in table 1. The specimens included 205 ovaries and 210 fallopian tubes.

\section{i. Definition of Gross Abnormality}

The first analysis involved a determination of how frequently a grossly normal ovary or fallopian tube is found to contain cancer after microscopic assessment, since this is directly 
relevant to the feasibility of representative sampling in this setting. Since the ovary frequently displays a variety of gross changes, we sought to calculate the performance of each gross abnormality in predicting a microscopically confirmed cancer in the adnexa, and which combination of gross abnormalities minimized the likelihood that a confirmed cancer will be missed. Accordingly, accuracy and related parameters for each individual gross abnormality (and combination of abnormalities) in predicting microscopically confirmed cancer were calculated and are outlined in table 2 . As expected, setting a requirement for multiple concurrent gross abnormalities maximized specificity but minimized sensitivity. For example, the presence of ovarian enlargement and mass lesion/nodules and any cystic change resulted in a specificity of $100 \%$ but a sensitivity of $20 \%$. Since the current analysis was geared towards the maximization of cancer identification, we selected the combination of gross abnormalities that maximized sensitivity, even if specificity was minimized. Ovaries with any cystic change and/or enlargement and/or a mass/nodular lesion yielded the highest sensitivity (69\%) and lowest specificity (68\%) of the entire analysis for microscopically confirmed cancer (table 2). Therefore, an ovary was considered to be grossly abnormal if it displayed any of those features. The same principle was applied to the fallopian tube. However, the only discernible gross abnormalities were in cases with mass lesions or nodules. As such, a fallopian tube was considered to be grossly abnormal if it was described as having mass lesions or nodules.

\section{ii. Grossly Occult Cancers}

Using the aforementioned definitions for gross abnormality, we identified 4 grossly occult cancers out of 148 ovaries (2.7\%), and 3 grossly occult cancers out of 187 fallopian tubes $(1.67 \%)$, table 3 . There were no patients with grossly occult cancers that involved both the ovary and fallopian tube. The only patient with bilateral adnexal involvement had a grossly occult cancer on the right ovary, and a grossly evident cancer on the left ovary. Therefore, adnexa that harbored grossly occult cancers were present in 7 (6.7\%) of 105 consecutive endometrial cancer patients, and was occult bilaterally in $6(5.7 \%)$ of the 105 patients. The grossly occult cancers were unifocal in all 4 ovarian cases and in 2 of the 3 fallopian tube cases. A grossly occult metastatic clear cell carcinoma in the fallopian tube is illustrated in figures $1 \mathrm{~A}$ and $1 \mathrm{~B}$. On average, the cancer deposits were significantly smaller in the grossly occult cases than in the grossly abnormal cases (ovary: $0.43 \mathrm{~cm}$ versus $2.95 \mathrm{~cm}$ respectively, $\mathrm{p}=0.038$, fallopian tube $0.19 \mathrm{~cm}$ versus $1.74 \mathrm{~cm}$ respectively, $\mathrm{p}=0.026$ ). Regarding organ sub-localization for the metastatic deposits, 3 of the ovarian cases were cortical, and 1 was hilar. The latter case, a metastatic endometrial serous carcinoma, is illustrated in figures $1 \mathrm{C}$ and 1D. All 3 tubal cases were mural, non-fimbrial, and non-intraluminal. Five (71.4\%) of the 7 grossly occult cancers were identified in representatively sampled organs (table 3)

\section{iii. Block Requirements}

An average of 1.19 and 4.9 blocks per ovary were required for representatively sampled and completely processed cases respectively. Parallel figures for the fallopian tubes were 1.06 and 2.01 blocks per fallopian tube respectively. For the ovaries, a total of 461 blocks were required for complete processing, as compared with 132 blocks for representative sampling. The difference (329) was divided by the total number of ovaries (205), yielding 1.6 as the number of additional blocks per ovary that was required for complete processing over 
representative sampling. This translates to 3.2 for the typical patient with 2 ovaries. For the fallopian tube, the differences were less stark: an identical analysis yielded 0.56 additional blocks per patient that was required for complete processing over representative sampling. By combining the aforementioned figures for the ovary and fallopian tubes, a total of 3.76 additional blocks per patient was required for complete processing of the adnexa over representative sampling of its organs.

\section{iv. Cost Analysis}

A cost analysis was performed of the 3.76 additional sections for completely processed cases. At $\$ 5.2$ per block, the cost of the 3.76 additional sections for each patient, prior to physician review of the slides, was estimated to be $\$ 19.528$. The added cost of physician review was estimated to be $\$ 6.04$ per patient ( $\$ 1.51 /$ minute $\times 4$ minutes). Thus the total cost of 3.76 additional sections was $\$ 25.568$ per patient.

Of the 105 patients, adnexal organs were totally and representatively processed in 47 and 58 patients respectively . 42 of the 47 totally processed cases were evaluated after a policy change requiring complete processing went into effect. Of the 7 patients with grossly occult cancers in the adnexa, only 5 of the cancers would be considered occult in a clinically significant fashion. In one case, as previously noted, the contralateral ovary had a grossly evident tumor, and metastatic disease was present at other locations. In another case, metastatic disease was found in regional lymph nodes. In both cases, the adnexal organs were representatively sampled.

Five patients were upstaged to stage IIIA solely because of adnexal involvement by grossly occult tumors. Two of the cases were completely processed and identified after the policy change to complete processing. The remaining 3 were representatively sampled and preceded the policy change. Of the 42 cases that were completely processed between our policy change and the end of the study period, 2 grossly occult cancers involving the adnexae and no other sites were identified. Accordingly, the policy change resulted in an additional labor and materials related cost of $\$ 1073.86(\$ 25.568 \times 42)$ over the study period.

\section{DISCUSSION}

The potential clinical implications of adnexal involvement by an otherwise uterus-confined endometrial cancer are very significant, and underscore the importance of pathologic review of the adnexal organs. The comprehensiveness of that review, however, must be balanced against the economic environment of clinical practice, especially since endometrial cancer is a relatively common cancer. To our knowledge, there have been no previous studies, and accordingly no evidence based guidelines, on how the adnexa should be processed when salpingo-oophorectomy specimens are received in the context of endometrial cancer staging. In the present study, we performed a multifaceted analysis of the issue with 2 major findings:

First, we documented the frequency of grossly occult cancer in the setting of endometrial cancer staging. Even when using the most expansive definition of ovarian gross abnormality, we still identified $2.7 \%$ of grossly normal ovaries and $1.6 \%$ of grossly normal 
fallopian tubes that were found to harbor microscopic cancers. More significantly, we estimated that this would have affected 6.7\% (7/105) of all patients. These figures can best be described as representing the risk of understaging that is theoretically associated with representative adnexal sampling, which practitioners should be aware of when grossing policies are being formulated. The notion that microscopic cancers in grossly normal small organs (such as the ovary or tube) are more likely to be identified if the organs are entirely versus representatively processed may appear to be intuitive, but could not be specifically proven in this study. Five (71\%) of the 7 grossly occult cancers were in the representatively sampled group. If the cancers in those 5 cases were truly microscopic, then their capture on representative sections may have been entirely random. Alternatively, they may have manifested as very subtle gross aberrations that the prosector did not document in the gross descriptions, but which he/she nonetheless sampled.

The converse corollary may also be true in reducing the capture of microscopic cancers in completely processed specimens, i.e. the possibility that the prosector pays less attention to subtle gross aberrations if they know the entire organ will be processed, and accordingly, less attention would be paid to the orientation of tissue sections as they are placed in the cassette. In that scenario, processing of the adnexal organs will be subject to the same sampling errors as colonic polyps, for example, where studies have shown that reembedding, reorientation and obtaining additional levels resulted in an additional diagnosis in up to $22 \%$ of initially non-diagnostic, endoscopically-apparent polyps $(17,18)$. Additionally, our policy change to routine complete processing did not result in a notable increase in the number of grossly occult cancers detected. Indeed, of the 42 patients whose specimens were completely processed between our policy change and the end of the study period, only 2 grossly occult cancers were identified that involved the adnexa and no other sites. This number is comparable to the number of cancers $(n=3)$ identified in the period that preceded the policy change. Although there is a theoretical risk of misdiagnosis associated with representative sampling, based on the 2 aforementioned findings (most grossly occult cancers identified in representatively sampled organs, and no increase in the detection of clinically significant, grossly occult cancers after our policy change to complete processing), our study does not provide evidentiary support for routine complete processing. However, this study is limited by the relatively small sample size, given the infrequency of the event (adnexal involvement in endometrial cancers).

Second, we performed a cost analysis to quantify any additional expenses that may be associated with routine complete processing. The significance of this analysis is related to the robustness of evidence that would be required to justify an extraordinarily exorbitant expense, and the ability to weigh several more factors if the expense was minor. We found that 3.76 additional blocks are required per patient for routine complete processing of the adnexa, and that the total labor-related costs for these blocks to be $\$ 25.568$. These were conservative monetary estimates that focused on labor, and did not include the cost of transport or storage of blocks. This analytic approach was taken because the costs associated with these factors are either minor or difficult to apply to a single unit. Additionally, opportunity costs (i.e., cost of activities as measured in terms of the value of the next best alterative that was not selected), were not considered. Reimbursement rates for Medicare 
and many insurance companies are generally more dependent on specimen type than the number of blocks generated. For the 42 patients seen after our policy change, the total cost was only $\$ 1073.86(\$ 25.568 \times 42)$. Extrapolated nationally, this cost would be entirely dependent on the number of endometrial cancer-related resections that are seen at a given institution, and the cost would be significantly higher at institutions that perform more resections. However, even if those costs are quadrupled for a given year (\$4295.44), that expense must be weighed against the significantly higher expenses that would be associated with even a single endometrial cancer recurrence (i.e. a stage IIIA endometrial cancer patient that was mistakenly classified as stage I due to a grossly occult, unsampled adnexal deposit, and who accordingly did not receive adjuvant chemotherapy). A recurrence may involve a biopsy, pathologic evaluation of said biopsy, radiologic studies, increased clinic visits, chemotherapy and/or radiotherapy. More importantly, the emotional and psychological toll that a recurrence may have on a patient recovering from cancer cannot be overstated.

In summary, we have performed a comparative analysis of whole tissue processing versus representative sampling for salpingo-oophorectomy specimens that are obtained for endometrial cancer staging. The findings in this study of a relatively small sample do not provide direct evidentiary support for routine complete processing. However, the expenses associated with complete processing are relatively minor when viewed in an institutional context and when balanced against the potential expenses of a recurrence. Furthermore, our study provides baseline data on whose basis evidence-based decisions can be made when grossing policies are being formulated.

\section{Acknowledgments}

The authors thank David W. Anderson, HT (ASCP) for his invaluable contributions to the cost analysis.

\section{REFERENCES}

1. Berney DM, Wheeler TM, Grignon DJ, et al. International Society of Urological Pathology (ISUP) Consensus Conference on Handling and Staging of Radical Prostatectomy Specimens. Working group 4: seminal vesicles and lymph nodes. Mod Pathol. 2011; 24:39-47. [PubMed: 20818343]

2. Siegel R, Naishadham D, Jemal A. Cancer statistics, 2012. CA Cancer J Clin. 2012; 62:10-29. [PubMed: 22237781]

3. Creasman WT, Odicino F, Maisonneuve P, et al. Carcinoma of the corpus uteri. Int J Gynaecol Obstet. 2003; 83:79-118. [PubMed: 14763170]

4. Creasman WT, Eddy GL. Recent advances in endometrial cancer. Semin Surg Oncol. 1990; 6:339_ 42. [PubMed: 2263809]

5. National Comprehensive Cancer Network. [Accessed 06/11/2012] NCCN Clinical Practice Guidelines in Oncology (NCCN Guidelines). Uterine Neoplasms. Version 22012. http:// www.ncen.org

6. Westra, WH.; Hruban, RH.; Phelps, TH.; Isacson, C. Ovary and Fallopian Tube. In: Westra, WH.; Hruban, RH.; Phelps, TH.; Isacson, C., editors. Surgical Pathology Dissection: An Illustrated Guide. 2nd Ed. Springer; New York: 2003. p. 160-165.

7. Garel L, Dubois J, Grignon A, Filiatrault D, Van Vliet G. US of the pediatric female pelvis: a clinical perspective. Radiographics. 2001; 21:1393-407. [PubMed: 11706212]

8. Robboy, SJ.; Kraus, FT.; Kurman, RJ. Gross description, processing, and reporting of gynecologic and Obstetric specimens. In: Kurman, RJ., editor. Blaustein's pathology of the female genital tract. 5th ed. Springer; New York: 2002. p. 1319-1346. 
9. Fleischer AC, McKee MS, Gordon AN, et al. Transvaginal sonography of postmenopausal ovaries with pathologic correlation. J Ultrasound Med. 1990; 9:637-44. [PubMed: 2258995]

10. Sladkevicius P, Valentin L, Marsál K. Transvaginal gray-scale and Doppler ultrasound examinations of the uterus and ovaries in healthy postmenopausal women. Ultrasound Obstet Gynecol. 1995; 6:81-90. [PubMed: 8535922]

11. Buesa RJ. Productivity standards for histology laboratories. Ann Diagn Pathol. 2010; 14:107-24. [PubMed: 20227016]

12. Garcia E, Bennett A, DeFranco M, et al. American Society for Clinical Pathology's 2010 Wage Survey of U.S. Clinical Laboratories. Lab Medicine. 2011; 42:141-146.

13. Gage JO. The AMA/Specialty Society RVS Update Committee (RUC). Bull Am Coll Surg. 1993; 78:7-10. [PubMed: 10126542]

14. American Clinical Laboratory Association. [Accessed 06/15/2012] Comments of the American Clinical Laboratory Association on the Medicare Program. Payment policies under the physician fee schedule and other revisions to part B for CY 2012 Proposed Rule. (CMS-1524-P). http:// www.acla.com

15. Nash JW, Niemann T, Marsh WL, Frankel WL. To step or not to step: an approach to clinically diagnosed polyps with no initial pathologic finding. Am J Clin Pathol. 2002; 117:419-23. [PubMed: 11888080]

16. Association of American Medical Colleges. [Accessed 06/12/2012] Careers in Medicine. https:// www.aamc.org/students/medstudents/cim/specialties/63714/cim_pub_pathology.html

17. Calhoun BC, Gomes F, Robert ME, Jain D. Sampling error in the standard evaluation of endoscopic colonic biopsies. Am J Surg Pathol. 2003; 27:254-7. [PubMed: 12548174]

18. Polydorides AD, Yantiss RK. Chasing colonic "polyps": features that predict underlying adenomas in initially nondiagnostic endoscopic biopsy specimens. Am J Clin Pathol. 2007; 127:409-14.

[PubMed: 17276941] 

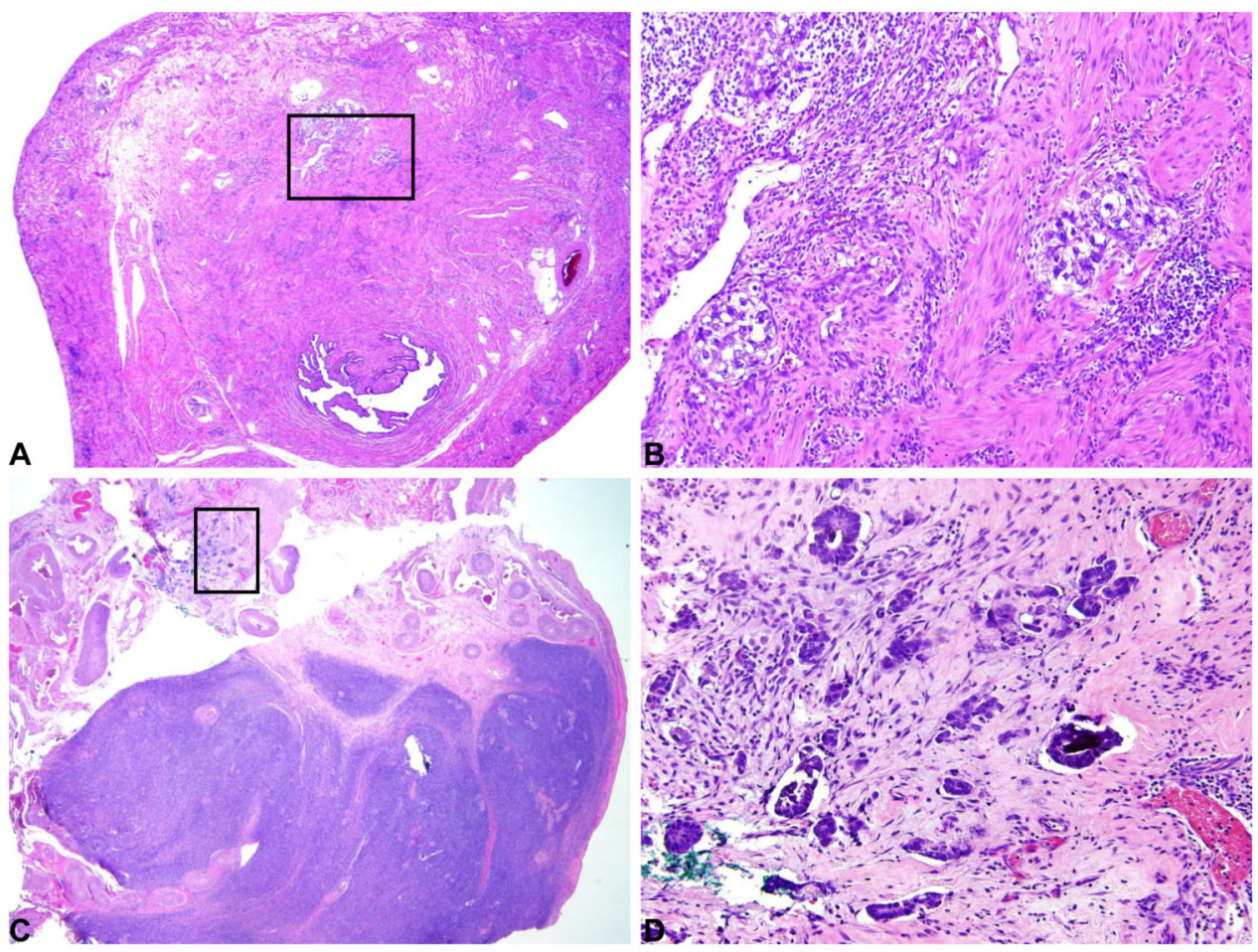

FIGURE 1.

A: Fallopian tube with a grossly occult, focal metastatic carcinoma (boxed inset), hematoxylin and eosin, original magnification $\times 20$.

B: Boxed inset from figure 1A, showing the focus of metastatic clear cell carcinoma, hematoxylin and eosin, original magnification $\times 200$.

C: Ovary with a grossly occult, focal metastatic carcinoma at the hilum (boxed inset), hematoxylin and eosin, original magnification $\times 20$.

D: Boxed inset from figure $1 \mathrm{C}$, showing the focus of metastatic serous carcinoma, hematoxylin and eosin, original magnification $\times 400$. 


\section{Table 1}

\section{Clinicopathologic information}

\begin{tabular}{|c|c|}
\hline Number of Patients & 105 \\
\hline \multicolumn{2}{|l|}{ Patient Age (years) } \\
\hline Mean \pm SD & $61.4 \pm 11.6$ \\
\hline Median & 62 \\
\hline Range & $26-81$ \\
\hline \multicolumn{2}{|c|}{ Histotype Distribution (carcinomas) } \\
\hline Serous & 6 \\
\hline Clear cell & 1 \\
\hline Endometrioid & 77 \\
\hline Endometrioid/Clear cell & 3 \\
\hline Endometrioid/Serous & 3 \\
\hline Endometrioid/Mucinous & 4 \\
\hline Carcinosarcoma & 9 \\
\hline Undifferentiated & 2 \\
\hline \multicolumn{2}{|l|}{ Grade Distribution $^{a}$} \\
\hline Grade I & 46 \\
\hline Grade II & 30 \\
\hline Grade III & 29 \\
\hline \multicolumn{2}{|l|}{ FIGO Stage Distribution } \\
\hline IA & 70 \\
\hline IB & 19 \\
\hline II & 2 \\
\hline IIIA & 5 \\
\hline IIIC1 & 3 \\
\hline IIIC2 & 1 \\
\hline IVB & 5 \\
\hline
\end{tabular}

${ }^{a}$ Carcinosarcomas, as well as all tumors with a $\geq 10 \%$ serous, clear cell, or undifferentiated carcinoma component were classified as grade III 
Table 2

Accuracy and related parameters for each gross abnormality

\begin{tabular}{|c|c|c|c|c|c|}
\hline & Sensitivity & Specificity & Accuracy & $\begin{array}{l}\text { Positive } \\
\text { Predictive Value }\end{array}$ & $\begin{array}{l}\text { Negative } \\
\text { Predictive Value }\end{array}$ \\
\hline \multicolumn{6}{|l|}{ OVARY } \\
\hline Enlargement & 38 & 91 & 87 & 26 & 94 \\
\hline Any cystic change & 50 & 82 & 79 & 19 & 95 \\
\hline Mass lesion/Nodules & 38 & 95 & 91 & 40 & 95 \\
\hline $\begin{array}{l}\text { Hemorrhage or } \\
\text { softening }\end{array}$ & 38 & 97 & 92 & 55 & 95 \\
\hline Simple cystic change & 19 & 82 & 81 & 8.3 & 92 \\
\hline Complex cystic change & 31 & 98 & 93 & 63 & 94 \\
\hline $\begin{array}{l}\text { Enlargement and any } \\
\text { cystic change }\end{array}$ & 40 & 97 & 93 & 44 & 96 \\
\hline $\begin{array}{l}\text { Enlargement and mass } \\
\text { lesion/nodules }\end{array}$ & 33 & 99 & 95 & 80 & 95 \\
\hline $\begin{array}{l}\text { Enlargement and mass } \\
\text { lesion/nodules and any } \\
\text { cystic change }\end{array}$ & 20 & 100 & 94 & 100 & 94 \\
\hline $\begin{array}{l}\text { Enlargement and mass } \\
\text { lesion/nodules and any } \\
\text { cystic change and } \\
\text { hemorrhage/softening }\end{array}$ & 22 & 100 & 94 & 100 & 95 \\
\hline $\begin{array}{l}\text { Any cystic change and } \\
\text { mass lesion/nodules }\end{array}$ & 29 & 100 & 95 & 100 & 95 \\
\hline $\begin{array}{l}\text { Simple cystic change } \\
\text { and enlargement }\end{array}$ & 6 & 98 & 91 & 20 & 92 \\
\hline $\begin{array}{l}\text { Complex cystic change } \\
\text { and mass } \\
\text { lesion/nodules }\end{array}$ & 25 & 100 & 94 & 100 & 94 \\
\hline $\begin{array}{l}\text { Complex cystic change } \\
\text { and enlargement }\end{array}$ & 19 & 99 & 93 & 75 & 94 \\
\hline $\begin{array}{l}\text { Any cystic change } \\
\text { and/or enlargement } \\
\text { and/or mass/nodular } \\
\text { lesion }\end{array}$ & 69 & 68 & 68 & 14 & 97 \\
\hline $\begin{array}{l}\text { Any cystic change } \\
\text { and/or enlargement } \\
\text { and/or mass/nodular } \\
\text { lesion and/or } \\
\text { hemorrhage/softening }\end{array}$ & 68 & 69 & 68 & 12 & 98 \\
\hline $\begin{array}{l}\text { Any cystic change or } \\
\text { mass/nodular lesion }\end{array}$ & 63 & 75 & 75 & 18 & 96 \\
\hline $\begin{array}{l}\text { Enlargement or mass } \\
\text { lesion }\end{array}$ & 50 & 91 & 87 & 33 & 95 \\
\hline $\begin{array}{l}\text { Any cystic change or } \\
\text { enlargement }\end{array}$ & 63 & 75 & 75 & 18 & 96 \\
\hline \multicolumn{6}{|l|}{$\begin{array}{l}\text { FALLOPIAN } \\
\text { TUBE }\end{array}$} \\
\hline Any cystic change & 0 & 97 & 95 & 0 & 97 \\
\hline Mass lesion/nodules & 40 & 96 & 95 & 22 & 98 \\
\hline
\end{tabular}




\begin{tabular}{|l|l|l|l|l|l|}
\hline & Sensitivity & Specificity & Accuracy & $\begin{array}{l}\text { Positive } \\
\text { Predictive Value }\end{array}$ & $\begin{array}{l}\text { Negative } \\
\text { Predictive Value }\end{array}$ \\
\hline $\begin{array}{l}\text { Any cystic change and } \\
\text { Mass lesion/nodules }\end{array}$ & 0 & 100 & 98 & 0 & 98 \\
\hline $\begin{array}{l}\text { Any cystic change or } \\
\text { Mass lesion/nodules }\end{array}$ & 40 & 93 & 92 & 13 & 98 \\
\hline
\end{tabular}

Gold standard: cancer as determined microscopically; Sensitivity True Positive (TP)/TP+False negative (FN); Specificity True negative(TN)/TN $+\mathrm{FP}$; Accuracy $\mathrm{TP}+\mathrm{TN} / \mathrm{TP}+\mathrm{TN}+\mathrm{False}$ positive $(\mathrm{FP})+\mathrm{FN}$; positive predictive value $\mathrm{TP} / \mathrm{TP}+\mathrm{FP}$; Negative predictive value $\mathrm{TN} / \mathrm{TN}+\mathrm{FN}$ 
Table 3

Pathologic data

\begin{tabular}{|c|c|c|}
\hline & OVARY & FALLOPIAN TUBE \\
\hline \multicolumn{3}{|l|}{ ALL CASES } \\
\hline Number & 205 & 210 \\
\hline \multicolumn{3}{|l|}{ Size $(\mathrm{cm})$} \\
\hline Maximal Dimension (mean length) $\pm \mathrm{SD}$ & $3.07 \pm 2.26$ & $5.5 \pm 1.7$ \\
\hline Median & 2.5 & 5.5 \\
\hline Range & $1.2-27$ & $1-10$ \\
\hline \multicolumn{3}{|l|}{ Sampling } \\
\hline Cases Representatively sampled (n) & 111 & 118 \\
\hline Blocks/Cassettes Required (total) & 132 & 125 \\
\hline Blocks/Cassettes Required per case & 1.19 & 1.06 \\
\hline Cases Entirely processed (n) & 94 & 92 \\
\hline Blocks/Cassettes Required (total) & 461 & 184 \\
\hline Blocks/Cassettes Required per case & 4.9 & 2 \\
\hline \multicolumn{3}{|l|}{ Blocks/Cassettes Required (n) } \\
\hline Mean \pm SD & $3.12 \pm 2.044$ & $1.595 \pm 1.16$ \\
\hline Median & 3 & 1 \\
\hline Range & $0.5-14$ & $0.5-7$ \\
\hline Microscopically confirmed cancer & 14 & 5 \\
\hline Cases representatively sampled (n) & 12 & 4 \\
\hline Cases entirely processed (n) & 2 & 1 \\
\hline Synchronous cancers in ovary and fallopian tube (n) & 0 & 0 \\
\hline \multicolumn{3}{|l|}{ GROSSLY ABNORMAL CASES ${ }^{a}$} \\
\hline Number & 57 & 23 \\
\hline Microscopically confirmed cancer & 10 & 2 \\
\hline Representatively sampled & 9 & 2 \\
\hline Entirely processed & 1 & 0 \\
\hline Size of Tumor in Ovary or Tube (Mean $\pm \mathrm{SD}, \mathrm{cm})$ & $2.95 \pm 1.56$ & $1.74 \pm 1.17$ \\
\hline \multicolumn{3}{|l|}{ Sampling (n) } \\
\hline Cases representatively sampled & 37 & 17 \\
\hline Cases entirely processed & 19 & 6 \\
\hline \multicolumn{3}{|l|}{ Blocks/Cassettes Required (n) } \\
\hline Mean \pm SD & $3.95 \pm 2.53$ & $1.5 \pm 1.33$ \\
\hline Median & 3 & 1 \\
\hline Range & $0.5-10$ & $0.5-7$ \\
\hline \multicolumn{3}{|l|}{ GROSSLY NORMAL CASES } \\
\hline Number & 148 & 187 \\
\hline
\end{tabular}




\begin{tabular}{|l|l|l|}
\hline & OVARY & FALLOPIAN TUBE \\
\hline Microscopically confirmed cancer & 4 & 3 \\
\hline Cases representatively sampled & 3 & 2 \\
\hline Cases entirely processed & 1 & 1 \\
\hline Size of Tumor in Ovary or Tube (Mean $\pm \mathrm{SD}, \mathrm{cm})$ & $0.43 \pm 0.21$ & $0.19 \pm 0.08$ \\
\hline Sampling (n) & & \\
\hline Cases Representatively sampled & 74 & 100 \\
\hline Cases Entirely processed & 74 & 87 \\
\hline Blocks/Cassettes Required (n) & & \\
\hline Mean \pm SD & $2.8 \pm 1.73$ & $1.6 \pm 1.14$ \\
\hline Median & 2 & 1 \\
\hline Range & $0.5-14$ & $0.5-6$ \\
\hline
\end{tabular}

${ }^{a}$ Grossly abnormal ovary: an ovary with enlargement and/or any cystic change and/or mass/nodular lesion; Grossly abnormal fallopian tube: a fallopian tube with any cystic change and/or mass/nodular lesion; SD standard deviation. 